The population diversity of $\mathrm{HBV}$ present in children failure to $H B$ vaccine and their mothers.

\section{PO-0260 FREQUENCY OF ANTIBIOTIC RESISTANCE PATTERNS IN BACTERIA ISOLATED FROM CHILDREN}

${ }^{1} \mathrm{R}$ Yousefimashouf, ${ }^{2} \mathrm{R}$ Esmaeili, ${ }^{3} \mathrm{MY}$ Alikhani, ${ }^{4} \mathrm{~A}$ Moshtaghi. ${ }^{1}$ Medical Microbiology, Hamadan University of Medical Sciences, Hamadan, Iran; ${ }^{2}$ Medicine, Hamadan University of Medical Sciences, Hamadan, Iran; ${ }^{3}$ Medical Microbiology, Hamadan University of Medical Sciences, Hamadan, Iran; ${ }^{4}$ Paediatrics, Hamadan University of Medical Sciences, Hamadan, Iran

\subsection{6/archdischild-2014-307384.910}

Background and objective Bacterial infectioins in particular meningitis, pneumonia and septicemia are still some of the most causes of mortalities in children. The aim of present study was to identify the most common bacterial agents causing infectionis in children under 14 and detection of antibiotic resistance paterns.

Material and methods During two years,1897 samples were obtained from the patients suspected bacterial infectioins. They were investigated for bacterial cultures, age, sex and antibiogram patterns. The species were identified by biochemical and serological methods.

Results Of 1897 samples, 563 (29.6\%) had positve bacterial culture. Of these $74.7 \%$ were gram negative and $25.3 \%$ gram positive. The most common species were Escherichia coli (34.1\%), Staphylococcus aureus (17.1\%), Psuedomonas aeroginosa (12.4\%), Kelebsiella (11\%) and Staphylococcus epidermidis (5.7\%). The most effective antibiotics against both gram positive and gram negative bacteria were ceftriaoxne, nitrofurantoin, nalidixic acid, amikacin and gentamycin.

Conclusion The gram negative bacteria in particular Escherichia coli, Psuedomonas aeroginosa and Kelebsiella are the predominant causes of bacterial infections in children under 14 in these regions. Most species showed a high relative resisitance to routine antibiotics such as ampicillin, trimethoprim and chloramphenicol.

\section{Infectious Diseases}

\section{P0-0260a EFFICACY OF MOBILE PHONE USE ON ADHERENCE TO NEVIRAPINE PROPHYLAXIS AND RETENTION IN CARE AMONG THE HIV-EXPOSED INFANTS IN PMTCT: A RANDOMISED CONTROLLED TRIAL}

L Kebaya, R Nduati, D Wamalwa, N Kariuki, A Bashir. Paediatrics, University of Nairobi, Nairobi, Kenya

\subsection{6/archdischild-2014-307384.911}

Background HIV is a major contributor to infant mortality. A significant gap remains between the uptake of infant and maternal antiretroviral regimens and only a minority of HIV-exposed infants receives prophylaxis and safe infant feeding. Losses to follow-up of HIV-exposed infants are associated with shortcomings of facility-based PMTCT models with weak community support of linkages. Use of mobile phones offers an opportunity to improving care and promoting retention for the mother-baby pairs, which is a major challenge in efforts to achieving an HIVfree generation.
Objectives To compare self-reported adherence to infant nevirapine (NVP) prophylaxis and retention in care in HIV exposed infants randomised to 2-weekly mobile phone call versus control (no phone calls).

Design Randomised controlled trial.

Methods 150 mother infant pairs were drawn from postnatal wards of 3 health facilities in Kisumu, and randomly assigned to receive either phone-based reminders on PMTCT messages or standard health care messages (no calls). The group in the intervention arm received phone calls fortnightly. Data on infant adherence to nevirapine, retention in care, safe infant feeding and early HIV testing among HIV-exposed infants were collected by use of a questionnaire at the scheduled Maternal Child Health $(\mathrm{MCH})$ visits (6 and 10 weeks). All analyses were intention to treat.

Results At 6 weeks follow-up, 90.7\% ( $\mathrm{n}=68$ ) of participants receiving phone calls reported adherence to infant NVP prophylaxis, compared with $72 \%(\mathrm{n}=54)$ of participants in the control group $(\mathrm{p}=0.005)$. Participants in the intervention arm were also significantly more likely to remain in care than participants in the control group $[78.7 \%(\mathrm{n}=59)$ vs. $58.7 \%(\mathrm{n}=44), \mathrm{p}=$ 0.009 at 6 weeks and $69.3 \%(\mathrm{n}=52)$ vs. $37.3 \%(\mathrm{n}=28), \mathrm{p}<$ 0.001 at 10 weeks].

Conclusions These results suggest that phone calls can be an important tool to improve adherence to infant NVP prophylaxis and retention in care for HIV-exposed infants.

\section{PO-0260b COMPARISON OF VESIKARI AND CLARK SEVERITY SCORES IN CHILDREN WITH ROTAVIRUS GASTROENTERITIS}

Z Kurugol, A Aslan, H Çetin, G Koturoglu. Pediatrics, Ege University Faculty of Medicine, Izmir, Turkey

\subsection{6/archdischild-2014-307384.912}

Two diarrhoea severity scales, the 20-point Vesikari scale and the 24-point Clark scale, are commonly used to assess the efficacy of rotavirus vaccines. However, the two scales have been compared previously in only a few studies by using the same patients.

The study was aimed to compare the Clark and Vesikari scales and to determine whether modified classifications would provide a better correlation between the two scales.

A total of 200 children with rotavirus gastroenteritis (RVGE) were evaluated. Of these, $57 \%$ were classified as severe by the Vesikari scale, while only $1.5 \%$ by the Clark scale $(\mathrm{p}<0.001)$. When the Clark 3-category scale was transformed into 2-category scale by merging mild and moderate categories as nonsevere, a good correlation with the Vesikari scale could not be found. Using the median of the severity scores as the severity threshold, could not provide a better correlation between the two scales either. Transforming the Vesikari 2-category scale into a 3 -category scale by further subdividing the severe category into two parts as moderate and severe $(\geq 16)$, thus using a score of 16 point as the severity threshold, provided a better correlation between the two severity scales, but still did not achieve a good level of agreement. Furthermore, still 89\% of all those with Vesikari score $\geq 16$ were classified as mild or moderate by the Clark scale.

The Clark and Vesikari severity scales differ significantly in the definition of severe RVGE. Even the reclassification attempts 